\title{
Prevalence of prediabetes and diabetes in children and adolescents with biopsy-proven nonalcoholic fatty liver disease
}

Short Title: Abnormal glucose tolerance in pediatric NAFLD

Valerio Nobili, $\mathrm{MD}^{1,2^{\star}}$, Alessandro Mantovani, $\mathrm{MD}^{3}$, Stefano Cianfarani, $\mathrm{MD}^{4,5}$, Anna Alisi, $\mathrm{PhD}^{6}$, Antonella Mosca, $\mathrm{MD}^{1}$, Maria Rita Sartorelli, $\mathrm{MD}^{7}$, Claudio Maffeis, $\mathrm{MD}^{8}$, Rohit Loomba, MD ${ }^{9}$, Christopher D. Byrne, MB BCh, PhD ${ }^{10,11}$, Giovanni Targher, MD ${ }^{3}$

\footnotetext{
${ }^{1}$ Hepatology, Gastroenterology and Nutrition Unit, IRCCS "Bambino Gesù" Children's Hospital, Rome, Italy

${ }^{2}$ Department of Pediatric, University "La Sapienza", Rome, Italy

${ }^{3}$ Section of Endocrinology, Diabetes and Metabolism, Department of Medicine, University and Azienda Ospedaliera Universitaria Integrata of Verona, Verona, Italy

${ }^{4}$ Dipartimento Pediatrico Universitario Ospedaliero "Bambino Gesù" Children's Hospital-IRCCS, "Tor Vergata” University, Rome, Italy

${ }^{5}$ Department of Women's and Children's Health, Karolinska Institute, Stockholm, Sweden

${ }^{6}$ Research Unit of Molecular Genetics of Complex Phenotypes, IRCCS “Bambino Gesù" Children's Hospital, Rome, Italy

${ }^{7}$ Hepato-Metabolic Disease Unit, "Bambino Gesù" Children's Hospital, IRCCS, Rome, Italy

${ }^{8}$ Pediatric Diabetes and Metabolic Disorders Unit, Department of Surgery, Dentistry, Pediatrics and Gynaecology, University Hospital of Verona, Verona, Italy

${ }^{9}$ NAFLD Research Center, Division of Gastroenterology, University of California, San Diego, CA, United States

${ }^{10}$ Nutrition and Metabolism, Faculty of Medicine, University of Southampton, Southampton, UK

${ }^{11}$ Southampton National Institute for Health Research Biomedical Research Centre, University Hospital Southampton, Southampton General Hospital, Southampton SO16 6YD, UK
}

${ }^{*}$ Deceased

Word count: abstract 248; text 4,487 (excluding title page, abstract, acknowledgments, references, tables and figure legend); n. 4 Tables $+n .1$ Figure $+\underline{n} .8$ supplementary Tables

Address for correspondence:

Prof. Giovanni Targher, MD

Section of Endocrinology, Diabetes and Metabolism

Department of Medicine

University and Azienda Ospedaliera Universitaria Integrata

Piazzale Stefani, 1

37126 Verona, Italy

Phone: +39-045-8123110

E-mail: giovanni.targher@univr.it 


\section{ACKNOWLEDGMENTS}

Conflicts of Interest: none.

Funding Sources: GT is supported in part by grants from the University School of Medicine of Verona, Verona, Italy. CDB is supported in part by grants from the Southampton National Institute for Health Research Biomedical Research Centre, Southampton, UK.

Authors' Contributions: VN, AA and GT conceived and designed the study. VN, AA, AM and $\mathrm{CM}$ researched data and reviewed/edited the manuscript. AM, SC, MRS, RL and CDB contributed to discussion and reviewed/edited the manuscript. GT and AM analyzed the data. GT wrote the manuscript. GT and AA are the guarantors of this work and, as such, had full access to all the data of the study and take responsibility for the integrity and accuracy of data. All authors approved the final version of the manuscript.

Keywords: glucose intolerance; prediabetes; diabetes; NAFLD; pediatrics 


\section{ABSTRACT}

Background \& Aims: We undertook a cross-sectional study of children/adolescents with and without non-alcoholic fatty liver disease (NAFLD) to compare the prevalence of prediabetes and diabetes, and to examine the role of abnormal glucose tolerance as a predictor of liver disease severity.

Methods: We recruited a cohort of 599 Caucasian children/adolescents with biopsyproven NAFLD, and 118 children/adolescents without NAFLD, who were selected to be similar for age, sex, body mass index and waist circumference to those with NAFLD. The diagnosis of prediabetes and diabetes was based on either hemoglobin A1c, fasting plasma glucose or 2-hour post-load glucose concentrations.

Results: Children/adolescents with NAFLD had a significantly higher prevalence of abnormal glucose tolerance (prediabetes or diabetes) than those without NAFLD (20.6\% vs. $11 \%, p=0.02)$. In particular, $124(20.6 \%)$ of children/adolescents with NAFLD had abnormal glucose tolerance, with $19.8 \%(n=119)$ satisfying the diagnostic criteria for prediabetes and $0.8 \%(n=5)$ satisfying the criteria for diabetes. The combined presence of prediabetes and diabetes was associated with a two-fold increased risk of non-alcoholic steatohepatitis (NASH; unadjusted-OR 2.19, 95\%Cl 1.47-3.29, p<0.001). However, this association was attenuated (but remained significant) after adjustment for age, sex, waist circumference (adjusted-OR 1.69, 95\% $\mathrm{Cl} 1.06-2.69, p=0.032$ ), and also the patatin-like phospholipase domain-containing protein-3 rs738409 polymorphism. These two latter variables were strongly associated with NASH.

Conclusions: Abnormal glucose tolerance (especially prediabetes) is highly prevalent among children/adolescents with biopsy-proven NAFLD. These children also have a higher risk of $\mathrm{NASH}$, though central adiposity is the factor that is most strongly associated with NASH. 


\section{LAY SUMMARY}

Children with biopsy-proven NAFLD have a higher prevalence of abnormal glucose tolerance (prediabetes or type 2 diabetes) than children without NAFLD. Children with biopsy-proven NAFLD and abnormal glucose tolerance also have a higher prevalence of $\mathrm{NASH}$ compared with those with normal glucose tolerance, though central adiposity is the factor that is most strongly associated with NASH. 


\section{INTRODUCTION}

With the growing prevalence of childhood obesity, pediatric non-alcoholic fatty liver disease (NAFLD) has emerged as the most common chronic liver disease in children and adolescents in Western countries (1-3). NAFLD is a spectrum of progressive liver disease that encompasses simple steatosis, non-alcoholic steatohepatitis (NASH), advanced fibrosis and, ultimately, cirrhosis $(3,4)$. Compelling evidence indicates that NAFLD also has serious health consequences outside of the liver and is strongly associated with an increased risk of cardiovascular disease and abnormal glucose tolerance (prediabetes and type 2 diabetes) (5-7).

Prediabetes and type 2 diabetes are two common conditions in adults with NAFLD (3,6-8). Furthermore, the presence of established diabetes among adults with NAFLD is a strong risk factor for the development of advanced fibrosis and cirrhosis, and is also a significant predictor of liver-related mortality $(3,6,8)$. To date, the impact of prediabetes and diabetes in children and adolescents with NAFLD has been less well defined. Although insulin resistance occurs almost universally in children/adolescents with $\operatorname{NAFLD}(1,2)$, the prevalence of prediabetes and diabetes in children/adolescents with biopsy-proven NAFLD is uncertain and, previously, the sample sizes of published studies have been usually too small to support a stable estimate of the prevalence of these two conditions in the pediatric NAFLD population (9-11). It is also uncertain whether abnormal glucose tolerance is a risk factor for NASH in children and adolescents. Recently, in a multi-ethnic cohort study of 675 United States (US) obese children/adolescents with biopsy-confirmed NAFLD enrolled in the NASH-Clinical Research Network, the authors reported that the prevalence of prediabetes and type 2 diabetes was $23.4 \%$ and $6.5 \%$, respectively (12). Moreover, children/adolescents with prediabetes or diabetes also had a greater prevalence of NASH compared to their counterparts with normal glucose tolerance (12). 
Given the unexpectedly high prevalence of abnormal glucose tolerance (especially diabetes) in this multi-ethnic cohort of US children/adolescents with NAFLD (12), we believe that it is important to have data from other countries. Therefore, we undertook a large, cross-sectional cohort study of Caucasian children/adolescents with biopsy-proven NAFLD and children/adolescents without NAFLD, who were selected to be comparable for age, sex, waist circumference and body mass index (BMI). The major aims of our study were: 1) to compare the prevalence of prediabetes and diabetes between children/adolescents with and without NAFLD; 2) to examine the differences in main clinical and biochemical parameters between children/adolescents with biopsy-confirmed NAFLD and abnormal glucose tolerance (diabetes and/or prediabetes) and their counterparts with normal glucose tolerance; and 3) to investigate the association between the presence of prediabetes/diabetes and liver histology characteristics.

\section{MATERIALS AND METHODS}

\section{Subjects}

We enrolled 599 unrelated Caucasian children and adolescents (298 boys and 301 girls; age 5-17 years) with an ultrasonographic diagnosis of severe hepatic steatosis or persistently ( $\geq 6$ months) elevated serum aminotransferase concentrations, who were referred to the Liver Unit of the "Bambino Gesù" Children's Hospital of Rome (Italy) between January 2003 and September 2018, and who accepted to undergo liver biopsy for the diagnosis and staging of NAFLD. This clinical practice is in agreement with the diagnostic flow chart proposed by the European Society for Pediatric Gastroenterology, Hepatology, and Nutrition (ESPGHAN) Hepatology Committee (13). All children were 
tested to exclude secondary causes of hepatic steatosis, such as alcohol consumption, total parenteral nutrition, and chronic use of drugs known to induce hepatic steatosis (e.g., valproate, amiodarone and prednisone). Hepatitis B and C, cytomegalovirus, Epstein-Barr virus infections and coeliac disease were excluded according to appropriate serological tests. Autoimmune liver disease, metabolic liver disease, Wilson's disease and alpha-1antitrypsin-associated liver disease were ruled out using standard clinical, laboratory and histological criteria. Children with previously diagnosed type 1 diabetes were excluded from the study. The study protocol was approved by the ethics committee of the "Bambino Gesù" Children's Hospital. Written informed consent was obtained from the parents of each child.

We also enrolled a sample of 118 obese children and adolescents (69 boys and 49 girls; age 5-17 years) without NAFLD, who were randomly selected to be similar for age, sex, $\mathrm{BMI}$ and waist circumference to children/adolescents with biopsy-proven NAFLD. These subjects were recruited from a subset of 347 children/adolescents without NAFLD on ultrasonography, who were included in a whole cohort of 514 (54.3\% male; mean age: $11.5 \pm 2.8$ years) Italian obese children and adolescents, who were referred to the obesity outpatient clinic of the Pediatric Diabetes and Metabolic Disorders Unit of the University Hospital of Verona (Italy), and who consecutively underwent liver ultrasonography for detecting NAFLD, after excluding those with specific genetic causes of obesity (e.g., Prader-Willi syndrome, Cohen syndrome), known chronic liver diseases (e.g., hepatitis by virus, drugs, autoimmunity, hemochromatosis and Wilson's disease), alcohol consumption and chronic use of any drugs (14). As part of the routine investigation of children in the obesity outpatient clinic, two experienced radiologists (who were blinded to the clinical details of participants) performed liver ultrasonography in all these children (14). Hepatic steatosis was diagnosed according to ultrasonographic characteristics, including diffuse 
hyper-echogenicity of the liver relative to the kidneys, ultrasonography beam attenuation, or poor visualization of the intrahepatic vessel borders and diaphragm $(4,14)$. The protocol of this study was approved by the local Ethics Committee of the University Hospital of Verona. Written informed consent was obtained from the parents of each child.

\section{Clinical and laboratory data}

BMI was measured as kilograms divided by the square of height in meters and then standardized BMI z-score was also calculated. Waist circumference was measured, with the patient in a standing position, on the horizontal plan between the lowest portion of the rib cage and the iliac crest. Blood pressure was measured in the right arm using a standard sphygmomanometer. Elevated blood pressure was defined by systolic or diastolic blood pressure $>95^{\text {th }}$ percentile for age and sex.

Venous blood samples were collected in the morning after an overnight fast. Measurements of serum glucose, aspartate aminotransferase (AST), alanine aminotransferase (ALT), gamma-glutamyltransferase (GGT) and other biochemical blood parameters were obtained using standard laboratory procedures at the central Laboratory of the hospital. Low-density lipoprotein (LDL)-cholesterol was calculated using the Friedewald's equation. Fasting insulin levels were measured by a chemiluminescent immunoassay. Hemoglobin $\mathrm{A} 1 \mathrm{c}(\mathrm{HbA} 1 \mathrm{c})$ was measured using a high-performance liquid chromatography analyzer. Homeostasis model assessment (HOMA-IR) score was used for estimating insulin resistance (15). A standard 2-hour oral glucose tolerance test (OGTT) was also performed.

\section{Definition of glucose tolerance status}


In accordance with the 2019 American Diabetes Association (ADA) recommendations for screening and diagnosis of both prediabetes and diabetes (16), children were considered to have prediabetes if they met at least one of the following three criteria: fasting glucose level between $100 \mathrm{mg} / \mathrm{dL}(5.6 \mathrm{mmol} / \mathrm{L})$ and $125 \mathrm{mg} / \mathrm{dL}(6.9 \mathrm{mmol} / \mathrm{L})$ or $\mathrm{HbA} 1 \mathrm{c}$ level between 39 and $47 \mathrm{mmol} / \mathrm{mol}$ (5.7-6.4\%) or 2-hour post-load glucose level between 140 $\mathrm{mg} / \mathrm{dL}$ and $199 \mathrm{mg} / \mathrm{dL}$ (7.8-11.0 mmol/L). Children were considered to have diabetes if they met at least one of the 3 criteria: fasting glucose level $\geq 126 \mathrm{mg} / \mathrm{dL}(\geq 7.0 \mathrm{mmol} / \mathrm{L})$ or $\mathrm{HbA} 1 \mathrm{c}$ level $\geq 48 \mathrm{mmol} / \mathrm{mol}(\geq 6.5 \%)$ or 2 -hour post-load glucose level $>200 \mathrm{mg} / \mathrm{dL}(\geq 11.1$ $\mathrm{mmol} / \mathrm{L})$. Children were considered to have normal glucose tolerance if neither the criteria for prediabetes nor diabetes were met (16).

\section{Liver histology}

Liver biopsies were performed using an automatic core biopsy 18-gauge needle (Biopince, Amedic, Sweden) under general anesthesia and ultrasound guidance, and then processed as described elsewhere (17). The main histological features commonly described for NAFLD, including hepatic steatosis, inflammation, hepatocyte ballooning and fibrosis, were scored according to the NASH Clinical Research Network scoring system, which has been validated in the pediatric population (18). Briefly, hepatic steatosis was graded on a fourpoint scale: (0) steatosis involving fewer than $5 \%$ of hepatocytes, (1) steatosis involving up to $33 \%$ of hepatocytes, (2) steatosis involving $33 \%$ to $66 \%$ of hepatocytes, and (3) steatosis involving more than $66 \%$ of hepatocytes. Lobular inflammation was graded on a four-point scale: (0) no foci, (1) fewer than two foci per $200 \times$ field, (2) two to four foci per $200 \times$ field, and (3) more than four foci per $200 \times$ field. Hepatocyte ballooning was graded from 0 to 2: (0) no balloon cells, (1) few balloon cells, and (2) many/prominent balloon cells. Portal tract inflammation was graded from 0 to 3 ( 0 is none, $1=$ mild, $2=$ moderate and $3=$ severe). The stage of hepatic fibrosis was quantified using a five-point scale: (0) no 
fibrosis, (1) perisinusoidal or periportal fibrosis [(1a) mild, zone 3, perisinusoidal; (1b) moderate, zone 3, perisinusoidal; and (1c) portal/periportal], (2) perisinusoidal and portal/periportal fibrosis, (3) bridging fibrosis, and (4) cirrhosis. Liver biopsies were diagnosed as definite NASH, borderline NASH, or NAFLD without NASH (i.e., no-NASH) based on the aggregate presence and degree of the individual features of NAFLD. In accord with the NASH Clinical Research Network (17), we calculated the NAFLD Activity Score (NAS), which is defined as the unweighted sum of the scores for steatosis, lobular inflammation, and ballooning; thus ranging from 0 to 8 . Liver fibrosis is not included as a component of this score. Cases with NAS of 0 to 2 were largely considered not diagnostic of $\mathrm{NASH}$; on the other hand, most cases with scores of $\geq 5$ were diagnosed as definite NASH. Cases with activity scores of 3 and 4 were diagnosed as indeterminate or borderline NASH (18). A typical set of minimum criteria to diagnose NASH would include at least $5 \%$ macro-vesicular steatosis, lobular inflammation and hepatocyte injury as manifested by ballooning degeneration $(12,18)$. Cases determined to be NAFLD without NASH showed at least $5 \%$ steatosis with no or minimal inflammation. This assignment of $\underline{\mathrm{NASH}}$, borderline NASH, or NAFLD without NASH was confirmed by a liver pathologist. In this study, a different grading histologic score for pediatric NAFLD was also used to test the reproducibility of our original results, i.e., the Pediatric NAFLD Histological Score (PNHS) (19), which is calculated by using the weighted sum of hepatic steatosis, ballooning, lobular inflammation and portal inflammation, according to the following formula: $\mathrm{PNHS}=100 \times \exp (\mathrm{zPNHS}) /[\exp (z \mathrm{PNHS})]$, where $z \mathrm{PNHS}=-8.4+2.5 \times$ steatosis $+3.5 \times$ ballooning $+3.4 \times$ lobular inflammation $+0.87 \times$ portal inflammation .

\section{PNPLA3 genotyping}

Blood samples were collected and the genomic DNA was extracted by QIAamp Blood MiniKit (Qiagen, Hilden, Germany), according to the manufacturer's instructions. The rate 
of success in extracting DNA was $100 \%$ for each study group. The PNPLA3 rs 738409 C>G SNP, encoding I148M, was genotyped with a 5'-nuclease TaqMan assay (Assay on Demand for rs738409, Applied Biosystems, Foster City, CA) by personnel unaware of the clinical status of the patients. Post-polymerase chain reaction allelic discrimination was carried out through the measurement of allele-specific fluorescence on the Opticon 2 detection system (MJ Research, Waltham, MA). Random samples were confirmed by direct genotyping (Applied Biosystems 3500 Genetic Analyzer), which provided concordant results in all cases; controls were included in all analyzed batches, and quality controls were used to verify the reproducibility of the results (20). Distribution of the genotype was in Hardy-Weinberg equilibrium and the call rate exceeded 99\%. PNPLA3 genotyping was available for all children/adolescents without NAFLD $(n=118)$ and for $328(54.8 \%)$ children/adolescents with biopsy-proven NAFLD, respectively.

\section{Statistical analysis}

Data are expressed as means $\pm S D$ or absolute and relative frequencies. Standard descriptive statistics (i.e., the Fisher's exact test or the chi-squared test for categorical variables, the one-way ANOVA for normally distributed continuous variables and the Kruskal-Wallis test for non-normally distributed variables) were used to compare the differences in main clinical and biochemical characteristics (Table 1), as well as in liver histology characteristics (Table 2) among children/adolescents with NAFLD, stratified by glucose tolerance status; in these analyses children/adolescents with abnormal glucose tolerance (i.e., prediabetes or diabetes) were combined into a single category, because the number of children with diabetes was small $(n=5)$. We believe that combining diabetes with prediabetes is legitimate, because diabetes/prediabetes are diagnosed by glucose levels (which only differ by a very small amount) and because our study is in children, (unlike a similar analysis in adults) those children with a diagnosis of diabetes will have 
had diabetes for a very short period of time - meaning that as a group they are more likely to be similar, than different, from the group with prediabetes. Using glucose tolerance status as the exposure variable, the odds of having NASH among children/adolescents with NAFLD was determined using logistic regression analysis with the presence of histologic NASH (i.e., definite NASH vs. no-NASH/borderline NASH) as the binary outcome (Table 3). We performed three forced-entry logistic regression models: the first model was unadjusted (Table 3, unadjusted model); the second model was adjusted for age, sex and waist circumference (adjusted model 1); and the third model was further adjusted for the PNPLA3 rs738409 variant (adjusted model 2). The same logistic regression analyses were also repeated either using a three-level model (stratifying the dependent variable as no-NASH, borderline NASH and definite NASH) or using the individual histologic features of NAFLD (i.e., severe steatosis, severe lobular inflammation, ballooning degeneration or significant fibrosis) as dependent variables in the aforementioned logistic regression models (Table 4). Covariates included in these multivariable regression models were selected as potential confounders based on their significance in univariable regression analyses or based on their biological plausibility. A pvalue $<0.05$ was considered statistically significant. Statistical analyses were performed using STATA software, version 14.2 (STATA, College Station, Texas, USA).

\section{RESULTS}

The clinical and biochemical parameters of the 599 Caucasian overweight or obese children/adolescents with biopsy-proven NAFLD and the 118 children/adolescents without ultrasound-defined NAFLD are summarized in Table 1. The two groups of children/adolescents were similar for the proportions of males and females in each group, age (mean 11.7 vs. 11.3 years), BMI (mean 27.2 vs. $27.7 \mathrm{~kg} / \mathrm{m}^{2}$ ) and waist circumference 
(mean 85.5 vs. $84.6 \mathrm{~cm}$ ). Children/adolescents with biopsy-proven NAFLD had significantly lower HDL-cholesterol and higher serum triglyceride and liver enzyme levels compared to those without NAFLD. Children/adolescents with and without NAFLD also differed in terms of distribution of the PNPLA3 genotype. In addition, and more importantly, children/adolescents with biopsy-proven NAFLD had a significantly greater prevalence of abnormal glucose tolerance $(20.7 \%$ vs. $11 \%$, respectively). In particular, the prevalence of normal and abnormal glucose tolerance (prediabetes/diabetes) among children with biopsy-proven NAFLD was as follows: normal glucose tolerance was present in $79.3 \%$ (475 of 599), prediabetes in $19.8 \%$ (119 of 599 ), and diabetes in $0.8 \%$ (5 of 599 ; all these 5 children had newly diagnosed diabetes), respectively. Conversely, the prevalence of normal and abnormal glucose tolerance in children/adolescents without NAFLD was as follows: normal glucose tolerance was present in $88.9 \%$ (105 of 118) and prediabetes in $11 \%$ (13 of 110$) ;$ none of these children had diabetes. In multivariable logistic regression analysis, after pooling children/adolescents with and without NAFLD ( $n=717)$, we found that presence of NAFLD (adjusted-odds ratio $1.98,95 \% \mathrm{Cl} 1.07-3.64, p=0.031$ ) was independently associated with risk of abnormal glucose tolerance (prediabetes/diabetes) $\underline{\text { after adjusting for age, sex and waist circumference. Increased waist circumference was }}$ another variable that was independently associated with risk of abnormal glucose tolerance (adjusted-odds ratio 1.02, 95\% Cl 1.01-1.03).

As shown in Fig. 1, the prevalence of prediabetes and diabetes among children/adolescents with biopsy-proven NAFLD was almost identical in boys and girls. Similarly, age, BMI, waist circumference, HOMA-IR score, serum liver enzymes, PNPLA3 genotypes and histologic features of NAFLD did not significantly differ between boys and girls (Supplementary Tables 1 and 2). 
As also shown in Table 1, when children/adolescents with biopsy-proven NAFLD were grouped by glucose tolerance status, the mean age for those with prediabetes/diabetes was slightly but significantly higher than those with normal glucose tolerance. Adiposity measures (BMI and waist circumference), blood pressure, fasting glucose and insulin levels, HbA1c, 2-hour post-load insulin and glucose levels sharply increased across glucose tolerance categories. Conversely, there were no significant differences in sex, serum transaminases, total cholesterol, HDL-cholesterol or LDL-cholesterol levels by glucose tolerance status. Similarly, no significant difference was found in the distribution of the PNPLA3 genotype between the two groups.

Table 2 shows the histologic features of NAFLD by glucose tolerance status. Among children/adolescents with NAFLD, definite NASH on histology was present in $29.9 \%$ of those with normal glucose tolerance (142 of 475 ) and $48.4 \%$ of those with prediabetes or diabetes (60 of 124) $(p<0.001)$. Children/adolescents with prediabetes/diabetes also had higher degrees of hepatic steatosis and lobular inflammation compared with those with normal glucose tolerance. No significant differences were observed in ballooning degeneration and fibrosis stage between the two groups of children.

Supplementary Table 3 summarizes the clinical and biochemical parameters of participants, stratified by NASH status. Prediabetes or diabetes was present in nearly $30 \%$ of children/adolescents with NASH (60 of 202) and in nearly 16\% (64 of 397) of those without NASH. In addition, children/adolescents with NASH were older and more likely to be centrally obese, hypertensive and insulin resistant (as reflected by greater HOMA-IR score and higher fasting and 2-hour post-load insulin concentrations) compared to those without NASH. They also had higher triglycerides, lower HDL-cholesterol and higher 
serum liver enzyme levels. Notably, children/adolescents with NASH also had a greater prevalence of $P N P L A 3 \mathrm{C} / \mathrm{G}$ and $\mathrm{G} / \mathrm{G}$ genotypes than those without NASH.

Table 3 shows the association between the presence of abnormal glucose tolerance (prediabetes and diabetes combined into a single category) and risk of $\mathrm{NASH}$, and the effect of adjustment for established risk factors. In univariable regression analysis (unadjusted model), the presence of prediabetes/diabetes was associated with a $\sim 2.2$-fold increased risk of NASH (unadjusted-OR 2.19, 95\% CI 1.47-3.29, $p<0.001$ ). However, this association was attenuated after adjustment for age, sex and waist circumference (adjusted-OR 1.69, 95\% Cl 1.06-2.69, $p=0.032$ ) (adjusted model 1); increased waist circumference was found to be the strongest predictor of NASH $(p<0.001)$. As a supplementary analysis (adjusted model 2), these results remained unchanged even after further adjustment for the PNPLA3 rs738409 variant; however, this latter regression model should be interpreted cautiously, because PNPLA3 genotyping was available only in 328 (54.8\%) children and adolescents. The results did not substantially change even when the same logistic regression analyses were repeated using a three-level regression model, stratifying the dependent variable as no-NASH, borderline $\mathrm{NASH}$ and definite $\mathrm{NASH}$, respectively (supplementary Table 4). Notably, in all the aforementioned multivariable regression models, increased waist circumference was the strongest predictor of NASH (along with the presence of risk allele (G) of rs738409 in PNPLA3 gene). Almost identical results were found even when we excluded children/adolescents with diabetes $(n=5)$ from the analysis (Supplementary Table 5), or when we adjusted the results for BMI, instead of waist circumference, or when we used the PNHS, instead of the NAS, for diagnosing NASH (data not shown). Finally, when we tested whether plasma glucose levels (included as a continuous measure) was a predictor for the severity of liver disease, we found that neither fasting plasma glucose (supplementary Table 6) nor 2-hour post-load glucose 
concentrations (supplementary Table 7) were significantly associated with NASH after adjusting for potential confounding factors.

Table 4 shows the association between prediabetes/diabetes status and risk of different histological features of NAFLD. In univariable regression analyses (unadjusted model), the presence of prediabetes/diabetes was significantly associated with an almost two-fold increased risk of having both severe hepatic steatosis $(p<0.005)$ and severe lobular inflammation $(p<0.005)$, and marginally associated with ballooning degeneration $(p=0.069)$, but not with significant hepatic fibrosis. These associations were attenuated after adjustment for age, sex and waist circumference (waist circumference being the variable that was most strongly associated with histological features of NAFLD). $\underline{\text { Similar }}$ results were found even when we excluded children/adolescents with diabetes $(n=5)$ from the analysis (Supplementary Table 8).

\section{DISCUSSION}

The main findings of our large mono-ethnic cohort study were that Caucasian children/adolescents with biopsy-proven NAFLD had a higher prevalence of abnormal glucose tolerance (i.e., prediabetes or diabetes) compared to children/adolescents without $\operatorname{NAFLD}(20.6 \%$ vs. $11 \%)$, who were similar for age, sex and measures of adiposity. In particular, $20.6 \%$ (124 of 599) of children/adolescents with biopsy-confirmed NAFLD had abnormal glucose tolerance, with the large majority of them (19.8\%; 119 children) satisfying the diagnostic criteria for prediabetes (i.e., impaired fasting glycaemia or impaired glucose tolerance) and only $0.8 \%$ ( 5 children) satisfying the diagnostic criteria for diabetes. The prevalence of abnormal glucose tolerance was equivalent for boys and girls, 
possibly because both sexes had comparable values of age, BMI, waist circumference, HOMA-IR, and prevalence of NASH. Furthermore, we found that children/adolescents with biopsy-proven NAFLD and abnormal glucose tolerance also had a higher prevalence of NASH compared with their counterparts with normal glucose tolerance. In univariable regression analysis, the presence of prediabetes/diabetes was associated with a $\sim 2$.2-fold increased risk of $\mathrm{NASH}$. However, this association was attenuated (but remained significant) after adjustment for age, sex and waist circumference. Furthermore, neither fasting plasma glucose nor 2-hour post-load glucose concentrations (included as a continuous measure in multivariable regression analyses) were significantly associated with NASH after adjusting for potential confounding factors. Interestingly, in our multivariable logistic regression analyses, both increased waist circumference and the presence of PNPLA3 rs738409 polymorphism (i.e., the major genetic variant associated with greater predisposition to NASH and progressive liver fibrosis) $(21,22)$ were each strongly and independently associated with NASH.

In our cohort of children/adolescents with NAFLD, we found a prevalence of abnormal glucose tolerance (especially diabetes) that was much lower than that reported in the multi-ethnic cohort of US children/adolescents with NAFLD enrolled in the NASH-Clinical Research Network (12). In fact, in the US cohort, the authors reported that nearly $30 \%$ of children/adolescents with NAFLD had prediabetes or diabetes (based on clinical history and/or fasting laboratory parameters, i.e., fasting glucose and $\mathrm{HbA} 1 \mathrm{c}$ levels). In particular, $23.4 \%$ of these children had prediabetes and $6.5 \%$ had type 2 diabetes (12). It is also important to note that these children did not undergo OGTT testing for diagnosing prediabetes and diabetes (12). Since the use of OGTT increases the sensitivity of case detection (16), it is reasonable to assume that the lack of OGTT data has partly underestimated the rate of prediabetes and diabetes in the US cohort. That said, we 
believe that the marked differences in the prevalence of prediabetes and, especially, diabetes observed between our cohort and the US cohort (that were well comparable for sample size) may reflect differences in: a) age (mean age 12.6 vs. 11.7 years in the US cohort and in our cohort, respectively); b) adiposity measures (mean BMI 32.5 vs. 27.2 $\mathrm{kg} / \mathrm{m}^{2}$, and mean waist circumference $104.5 \mathrm{vs} .85 .5 \mathrm{~cm}$ in the US cohort and in our cohort, respectively) and c) race/ethnicity (65.9\% Hispanic/28.3\% non-Hispanic White/5.8\% Non-Hispanic in the US cohort vs. 100\% Caucasian in our cohort). In addition, the much higher prevalence of diabetes in the US cohort might also account in part for the more pronounced association seen between prediabetes/diabetes and the histological severity of NAFLD, compared to our cohort. In fact, the prevalence of advanced liver fibrosis was markedly higher in the US cohort compared with our cohort (fibrosis stage 3 : $15 \%$ vs. $0.8 \%$ ). As also highlighted by the same authors (12), the prevalence of diabetes in the US cohort appeared to be much higher than would be expected based on the contribution from obesity alone. Indeed, the SEARCH for Diabetes in Youth Study (i.e., a large observational study conducting population-based ascertainment of physiciandiagnosed diabetes in US youth) showed that the overall prevalence estimate for diabetes in children and adolescents in the United States was approximately $0.20 \%(23)$. Interestingly, in our study we also found that the prevalence of prediabetes/diabetes was nearly twice as high in children/adolescents with biopsy-proven NAFLD than in children/adolescents without NAFLD (20.6\% vs. $11 \%)$. The prevalence of abnormal glucose tolerance in our children without NAFLD was essentially superimposable on that observed in other Italian pediatric cohorts that also used OGTT testing for diagnosing prediabetes and diabetes. In fact, in these cohorts, involving obese children and adolescents, the prevalence rates of prediabetes and type 2 diabetes were nearly $8-10 \%$ and $<0.2 \%$, respectively (24-26). 
A major finding in our study was that children/adolescents with NAFLD and abnormal glucose tolerance had $\sim 2.2$ times the odds for NASH compared to their counterparts with normal glucose tolerance. They also had more severe hepatic steatosis and lobular inflammation. Furthermore, in the subset of 328 children with biopsy-proven NAFLD and PNPLA3 genotyping data, the strong association we observed between waist circumference and the risk of NASH persisted even after further adjustment for the PNPLA3 rs738409 polymorphism, which is the major genetic variant associated with greater predisposition to NASH and progressive liver fibrosis both in adults and children $(21,22,27)$. However, it should also be noted that in our multivariable regression analyses we combined the presence of prediabetes and diabetes (abnormal glucose tolerance) into a single category, because only 5 children had diabetes. Therefore, our database does not permit us to examine the differential impact of prediabetes versus diabetes on risk of $\mathrm{NASH}$. It is well known that NAFLD is highly prevalent in adults with type 2 diabetes (occurring in up to $70 \%$ of these individuals), and that patients with type 2 diabetes and NAFLD are also more likely (than patients with just NAFLD) to develop the more severe histologic forms of NAFLD (i.e., NASH, advanced fibrosis and cirrhosis), which can ultimately lead to hepatocellular carcinoma $(3,6-8)$.

Our study has both strengths and limitations that deserve further mention. First, the singlecenter, cross-sectional design of our study does not allow for establishing the temporality and causality of the observed associations. Second, similarly to other large cohort studies, we assigned our case definitions for prediabetes and diabetes on a single-time fasting laboratory measurement based on parameters (i.e., fasting glucose and $\mathrm{HbA} 1 \mathrm{c}$ levels), recommended by the ADA guidelines. However, most of our children and adolescents also underwent OGTT testing, which is a sensitive and accurate test for diagnosing prediabetes (impaired glucose tolerance) and diabetes (16). Therefore, we consider that the 
prevalence estimates of both prediabetes and diabetes are a reliable estimate of the true prevalence in our cohort of children with biopsy-confirmed NAFLD. Third, since we excluded patients with established type 1 diabetes, it is reasonable to assume that the majority of our children/adolescents had type 2 diabetes. However, we did not perform genetic testing for diagnosing other less common subtypes of diabetes (e.g., monogenic diabetes due to MODY mutations) $(16,28)$. Finally, since we studied a cohort of Caucasian (European) children and adolescents with biopsy-proven NAFLD, our findings might not be necessarily generalizable to other ethnic pediatric populations.

In conclusion, our large cohort study shows that abnormal glucose tolerance (especially prediabetes) was highly prevalent in Caucasian children/adolescents with wellcharacterized, biopsy-proven NAFLD, and this metabolic abnormality was much more frequent than in children/adolescents without NAFLD, who were similar for age, sex and measures of adiposity. Children with biopsy-proven NAFLD and abnormal glucose tolerance also had a higher risk of NASH compared with their counterparts with normal glucose tolerance. Furthermore, these results further highlight the role of central adiposity as the strongest predictor of NASH. Therefore, our findings further emphasize the importance of lifestyle modifications to improve diet and increase physical activity, as the first-line treatment for all children/adolescents with NAFLD not only for improving liver disease severity, but also for reducing obesity-related metabolic complications (such as abnormal glucose tolerance) in childhood and adolescence. 


\section{REFERENCES}

1. Fleet SE, Lefkowitch JH, Lavine JE. Current concepts in pediatric nonalcoholic fatty liver disease. Gastroenterol Clin North Am. 2017;46:217-231.

2. Mann JP, Valenti L, Scorletti E, Byrne CD, Nobili V. Nonalcoholic fatty liver disease in children. Semin Liver Dis. 2018;38:1-13.

3. Younossi Z, Anstee QM, Marietti M, Hardy T, Henry L, Eslam M, George J, Bugianesi E. Global burden of NAFLD and NASH: trends, predictions, risk factors and prevention. Nat Rev Gastroenterol Hepatol. 2018;15:11-20.

4. Byrne CD, Patel J, Scorletti E, Targher G. Tests for diagnosing and monitoring non-alcoholic fatty liver disease in adults. BMJ. 2018;362:k2734.

5. Selvakumar PKC, Kabbany MN, Nobili V, Alkhouri N. Nonalcoholic fatty liver disease in children: hepatic and extrahepatic complications. Pediatr Clin North Am. 2017;64:659-675.

6. Byrne CD, Targher G. NAFLD: a multisystem disease. J Hepatol 2015;62:S47S64.

7. Adams LA, Anstee QM, Tilg H, Targher G. Non-alcoholic fatty liver disease and its relationship with cardiovascular disease and other extra-hepatic diseases. Gut. 2017;66:1138-1153.

8. Targher G, Lonardo A, Byrne CD. Nonalcoholic fatty liver disease and chronic vascular complications of diabetes mellitus. Nat Rev Endocrinol. 2018;14:99-114.

9. Schwimmer JB, Deutsch R, Rauch JB, Behling C, Newbury R, Lavine JE. Obesity, insulin resistance, and other clinicopathological correlates of pediatric nonalcoholic fatty liver disease. J Pediatr. 2003;143:500-505.

10. Cali AM, De Oliveira AM, Kim H, Chen S, Reyes-Mugica M, Escalera S, Dziura J, Taksali SE, Kursawe R, Shaw M, Savoye M, Pierpont B, Constable RT, Caprio $S$. Glucose dysregulation and hepatic steatosis in obese adolescents: is there a link? Hepatology. 2009;49:1896-903.

11.Patton HM, Yates K, Unalp-Arida A, Behling CA, Huang TT, Rosenthal P, Sanyal AJ, Schwimmer JB, Lavine JE. Association between metabolic syndrome and liver histology among children with nonalcoholic fatty liver disease. Am J Gastroenterol. 2010;105:2093-2102.

12. Newton KP, Hou J, Crimmins NA, Lavine JE, Barlow SE, Xanthakos SA, Africa J, Behling C, Donithan M, Clark JM, Schwimmer JB; Nonalcoholic Steatohepatitis Clinical Research Network. Prevalence of prediabetes and type 2 diabetes in children with nonalcoholic fatty liver disease. JAMA Pediatr. 2016;170:e161971. 
13. Vajro P, Lenta S, Socha P, Dhawan A, McKiernan P, Baumann U, Durmaz O, Lacaille F, McLin V, Nobili V. Diagnosis of nonalcoholic fatty liver disease in children and adolescents: position paper of the ESPGHAN Hepatology Committee. J Pediatr Gastroenterol Nutr. 2012;54:700-713.

14.Zusi C, Mantovani A, Olivieri F, Morandi A, Corradi M, Miraglia del Giudice E, Dauriz M, Valenti L, Byrne CD, Targher G, Maffeis C. Contribution of a genetic risk score to clinical prediction of hepatic steatosis in obese children and adolescents. Dig Liver Dis. 2019 in press.

15. Nobili V, Manco M, Ciampalini P, Diciommo V, Devito R, Piemonte F, Comparcola D, Guidi R, Marcellini M. Leptin, free leptin index, insulin resistance and liver fibrosis in children with non-alcoholic fatty liver disease. Eur $\mathrm{J}$ Endocrinol. 2006;155:735-743.

16. American Diabetes Association. Classification and diagnosis of diabetes: standards of medical care in diabetes-2019. Diabetes Care. 2019;42(Suppl 1):S13-S28.

17. Manco M, Bedogni G, Marcellini M, Devito R, Ciampalini P, Sartorelli MR, Comparcola D, Piemonte F, Nobili V. Waist circumference correlates with liver fibrosis in children with non-alcoholic steatohepatitis. Gut. 2008; 57:1283-1287.

18. Kleiner DE, Brunt EM, Van Natta M, Behling C, Contos MJ, Cummings OW, Ferrell LD, Liu YC, Torbenson MS, Unalp-Arida A, Yeh M, McCullough AJ, Sanyal AJ; Nonalcoholic Steatohepatitis Clinical Research Network. Design and validation of a histological scoring system for nonalcoholic fatty liver disease. Hepatology. 2005;41:1313-1321.

19. Alkhouri N, De Vito R, Alisi A, Yerian L, Lopez R, Feldstein AE, Nobili V. Development and validation of a new histological score for pediatric nonalcoholic fatty liver disease. J Hepatol. 2012;57:1312-1318.

20. Valenti L, Alisi A, Galmozzi E, Bartuli A, Del Menico B, Alterio A, Dongiovanni P, Fargion S, Nobili V. I148M patatin-like phospholipase domain-containing 3 gene variant and severity of pediatric nonalcoholic fatty liver disease. Hepatology. 2010;52:1274-1280.

21. Romeo S, Kozlitina J, Xing C, Pertsemlidis A, Cox D, Pennacchio LA, Boerwinkle E, Cohen JC, Hobbs HH. Genetic variation in PNPLA3 confers susceptibility to nonalcoholic fatty liver disease. Nat Genet. 2008;40:1461-1465.

22. Anstee QM, Seth D, Day CP. Genetic factors that affect risk of alcoholic and nonalcoholic fatty liver disease. Gastroenterology. 2016;150:1728-1744. 
23. SEARCH for Diabetes in Youth Study Group, Liese AD, D'Agostino RB Jr, Hamman RF, Kilgo PD, Lawrence JM, Liu LL, Loots B, Linder B, Marcovina S, Rodriguez B, Standiford D, Williams DE. The burden of diabetes mellitus among US youth: prevalence estimates from the SEARCH for Diabetes in Youth Study. Pediatrics. 2006;118:1510-1518.

24. Maffeis C, Pinelli L, Brambilla P, Banzato C, Valzolgher L, Ulmi D, Di Candia S, Cammarata B, Morandi A. Fasting plasma glucose (FPG) and the risk of impaired glucose tolerance in obese children and adolescents. Obesity (Silver Spring). 2010;18:1437-1442.

25. Morandi A, Maschio M, Marigliano M, Miraglia Del Giudice E, Moro B, Peverelli $P$, Maffeis $C$. Screening for impaired glucose tolerance in obese children and adolescents: a validation and implementation study. Pediatr Obes. 2014;9:17-25.

26. Di Bonito P, Pacifico L, Chiesa C, Valerio G, Miraglia Del Giudice E, Maffeis C, Morandi A, Invitti C, Licenziati MR, Loche S, Tornese G, Franco F, Manco M, Baroni MG; "CARdiometabolic risk factors in overweight and obese children in ITALY" (CARITALY) Study Group. Impaired fasting glucose and impaired glucose tolerance in children and adolescents with overweight/obesity. J Endocrinol Invest. 2017;40:409-416.

27. Seko Y, Yamaguchi K, Itoh Y. The genetic backgrounds in nonalcoholic fatty liver disease Clin J Gastroenterol. 2018;11:97-102.

28. Shields BM, Hicks S, Shepherd MH, Colclough K, Hattersley AT, Ellard S. Maturity-onset diabetes of the young (MODY): how many cases are we missing? Diabetologia. 2010;53:2504-2508. 
Table 1. Main clinical and biochemical characteristics of children/adolescents with biopsy-proven NAFLD and control children/adolescents without NAFLD, as well as between children/adolescents with biopsy-proven NAFLD, stratified by glucose tolerance status.

\begin{tabular}{|c|c|c|c|c|c|c|}
\hline & $\begin{array}{l}\text { Children with } \\
\text { biopsy-proven } \\
\text { NAFLD ( } n=599)\end{array}$ & $\begin{array}{l}\text { Children without } \\
\text { NAFLD }(n=118)\end{array}$ & $\begin{array}{l}P \\
\text { value* }\end{array}$ & $\begin{array}{l}\text { Children with biopsy- } \\
\text { proven NAFLD and } \\
\text { normal glucose } \\
\text { tolerance }(n=475)\end{array}$ & $\begin{array}{l}\text { Children with biopsy- } \\
\text { proven NAFLD and } \\
\text { prediabetes/diabetes } \\
(n=124)\end{array}$ & $\begin{array}{l}P \\
\text { value }^{\star *}\end{array}$ \\
\hline Age (years) & $11.7 \pm 3.1$ & $11.3 \pm 2.5$ & 0.16 & $11.5 \pm 3.0$ & $12.6 \pm 3.2$ & $<0.001$ \\
\hline Sex (male), \% (n) & $49.7(298)$ & $58.5(69)$ & 0.12 & $48.8(232)$ & $53.2(66)$ & 0.29 \\
\hline Weight $(\mathrm{kg})$ & $63.2 \pm 22$ & $62.7 \pm 15.7$ & 0.82 & $61.2 \pm 20$ & $70.9 \pm 26$ & $<0.001$ \\
\hline BMl $\left(\mathrm{kg} / \mathrm{m}^{2}\right)$ & $27.2 \pm 5.4$ & $27.7 \pm 3.3$ & 0.35 & $26.8 \pm 5.1$ & $28.9 \pm 6.3$ & $<0.001$ \\
\hline Waist circumference $(\mathrm{cm})$ & $85.5 \pm 14$ & $84.6 \pm 9.2$ & 0.59 & $84.5 \pm 13$ & $89.2 \pm 15$ & $<0.005$ \\
\hline Systolic blood pressure $(\mathrm{mmHg})$ & $112 \pm 13$ & $114 \pm 13$ & 0.18 & $110 \pm 12$ & $117 \pm 13$ & $<0.001$ \\
\hline Diastolic blood pressure $(\mathrm{mmHg})$ & $65 \pm 10$ & $66 \pm 9$ & 0.25 & $64 \pm 10$ & $67 \pm 10$ & $<0.005$ \\
\hline $\mathrm{HbA} 1 \mathrm{c}(\mathrm{mmoL} / \mathrm{mol})$ & $33 \pm 5$ & $35 \pm 4$ & 0.12 & $31 \pm 3$ & $38 \pm 5$ & $<0.001$ \\
\hline Fasting glucose (mg/dL) & $83 \pm 11$ & $81 \pm 8$ & 0.51 & $81 \pm 9$ & $88 \pm 15$ & $<0.001$ \\
\hline Fasting insulin (mlU/L) & $16.9 \pm 10.8$ & $18.4 \pm 13.8$ & 0.21 & $14.7 \pm 7.5$ & $25.6 \pm 16.1$ & $<0.001$ \\
\hline HOMA-insulin resistance score & $3.6 \pm 2.3$ & $3.5 \pm 2.8$ & 0.44 & $2.9 \pm 1.5$ & $5.5 \pm 3.5$ & $<0.001$ \\
\hline 2-hour OGTT glucose (mg/dL) & $110 \pm 24$ & $107 \pm 17$ & 0.63 & $104 \pm 17$ & $131 \pm 31$ & $<0.001$ \\
\hline 2-hour OGTT insulin (mIU/L) & $106 \pm 89$ & $86 \pm 66$ & 0.07 & $90.8 \pm 70$ & $158 \pm 121$ & $<0.001$ \\
\hline Total cholesterol $(\mathrm{mg} / \mathrm{dL})$ & $159 \pm 32$ & $162 \pm 29$ & 0.34 & $159 \pm 33$ & $160 \pm 30$ & 0.76 \\
\hline LDL cholesterol (mg/dL) & $99 \pm 29$ & $95 \pm 26$ & 0.12 & $99 \pm 29$ & $101 \pm 29$ & 0.44 \\
\hline HDL cholesterol (mg/dL) & $46 \pm 12$ & $49 \pm 11$ & $<0.05$ & $47 \pm 12$ & $44 \pm 13$ & 0.06 \\
\hline Triglycerides $(\mathrm{mg} / \mathrm{dL})$ & $109 \pm 64$ & $89 \pm 44$ & $<0.01$ & $106 \pm 64$ & $123 \pm 66$ & $<0.01$ \\
\hline AST (IU/L) & $38 \pm 23$ & $22 \pm 6.8$ & $<0.001$ & $37 \pm 23$ & $40 \pm 23$ & 0.28 \\
\hline ALT (IU/L) & $53 \pm 49$ & $24 \pm 13$ & $<0.001$ & $51 \pm 49$ & $58 \pm 49$ & 0.18 \\
\hline GGT (IU/L) & $23 \pm 16$ & NA & NA & $22 \pm 15$ & $27 \pm 18$ & $<0.01$ \\
\hline Normal glucose tolerance, \% (n) & $79.3(475)$ & $88.9(105)$ & 0.02 & & & \\
\hline Prediabetes/diabetes, \% (n) & $20.7(124)$ & $11.0(13)$ & & & & \\
\hline PNPLA3 rs738409 variant & $n=328$ & $n=118$ & $<0.001$ & $n=256$ & $n=71$ & 0.29 \\
\hline CC genotype, \% (n) & $30.8(101)$ & $60.2(71)$ & & $31.3(80)$ & $29.2(21)$ & \\
\hline GC genotype, $\%(n)$ & $43.0(141)$ & $35.6(42)$ & & $44.5(114)$ & $37.5(27)$ & \\
\hline GG genotype, $\%(n)$ & $26.2(86)$ & $4.2(5)$ & & $24.2(62)$ & $33.3(24)$ & \\
\hline
\end{tabular}

GG genotype, \% (n)

Data are expressed as means \pm SD or relative percentages (absolute percentages are reported in parenthesis). Data on 2-hour post-OGTT glucose levels and HbA1c were available in 499 and 345 children/adolescents with biopsy-proven NAFLD, respectively. Children/adolescents with prediabetes or diabetes were combined into a single category, because the number of subjects with diabetes was variables, . 'Differences between children/adolescents with and without NAFLD were tested by the Fisher's exact test for categorical variables, the Student's t-test for normally distributed continuous variables, and the Mann-Whitney test for non-normally distributed continuous variables (i.e., fasting insulin, 2-hour post-OGTT insulin, HOMA-IR score, triglycerides and liver enzymes). ${ }^{\star *}$ Difference
between the two groups of children/adolescents with NAFLD, stratified by glucose metabolism status were tested by the Fisher's exact test for categorical variables, the Student's t-test for normally distributed continuous variables, and the Mann-Whitney U test for non-normally distributed continuous variables (i.e., fasting insulin, 2-hour post-OGTT insulin, HOMA-IR score, triglycerides and liver enzymes), respectively. 


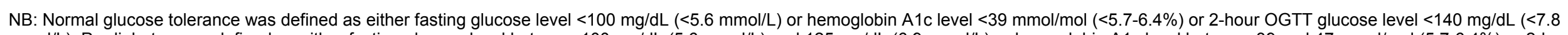

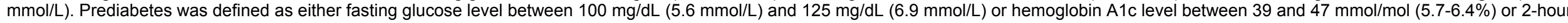
OGTT glucose level between $140 \mathrm{mg} / \mathrm{dL}(7.8 \mathrm{mmol} / \mathrm{L})$ and $199 \mathrm{mg} / \mathrm{dL}(11.0 \mathrm{mmol} / \mathrm{L})$. Type 2 diabetes was defined as either fasting glucose level at least $126 \mathrm{mg} / \mathrm{dL}(7.0 \mathrm{mmol} / \mathrm{L})$ or hemoglobin A1c level at least $48 \mathrm{mmol} / \mathrm{mol}(6.5 \%)$ or 2-hour OGTT glucose level at least $200 \mathrm{mg} / \mathrm{dL}(>11.0 \mathrm{mmol} / \mathrm{L})$

Abbreviations: ALT, alanine aminotransferase, AST, aspartate aminotransferase; BMI, body mass index; GGT, gamma-glutamyltransferase; HbA1c, hemoglobin A1c; HOMA-IR, homeostasis model assessment-insulin resistance; NA, not available; OGTT, oral glucose tolerance test; PNPLA3, patatin-like phospholipase domain-containing protein 3. 
Table 2. Liver histology characteristics of children/adolescents with biopsy-proven NAFLD, stratified by glucose tolerance status.

\begin{tabular}{|c|c|c|c|c|}
\hline & $\begin{array}{l}\text { Whole } \\
\text { sample } \\
(n=599)\end{array}$ & $\begin{array}{l}\text { Normal glucose } \\
\text { tolerance } \\
(n=475)\end{array}$ & $\begin{array}{l}\text { Prediabetes/ } \\
\text { diabetes status } \\
(n=124)\end{array}$ & $P$ value \\
\hline Steatosis grade, \% (n) & & & & $<0.005$ \\
\hline$<5 \%$ & $10.8(65)$ & $12(57)$ & $6.4(8)$ & \\
\hline $5 \%-33 \%$ & $31.4(188)$ & $33.5(159)$ & $23.4(29)$ & \\
\hline $33 \%-66 \%$ & $35.9(215)$ & $35.4(168)$ & $37.9(47)$ & \\
\hline$>66 \%$ & $21.9(131)$ & $19.1(91)$ & $32.3(40)$ & \\
\hline Lobular inflammation, \% (n) & & & & $<0.005$ \\
\hline$<2$ foci per $200 x$ field & $33.9(203)$ & $35.6(169)$ & $27.4(34)$ & \\
\hline $2-4$ foci per $200 \times$ field & $50.8(304)$ & $51.6(245)$ & $47.6(59)$ & \\
\hline$>4$ foci per $200 \times$ field & $15.3(92)$ & $12.8(61)$ & $25.0(31)$ & \\
\hline Ballooning, \% (n) & & & & 0.20 \\
\hline None & $40.6(243)$ & $41.5(197)$ & $37.1(46)$ & \\
\hline Few & $40.7(244)$ & $41.2(196)$ & $38.7(48)$ & \\
\hline Many & $18.7(112)$ & $17.3(82)$ & $24.2(30)$ & \\
\hline Fibrosis stage, \% (n) & & & & 0.26 \\
\hline No fibrosis & $48.3(284)$ & $50.1(234)$ & $40.3(50)$ & \\
\hline Perisinusoidal fibrosis & $53.3(260)$ & $42.8(200)$ & $50.8(63)$ & \\
\hline Periportal fibrosis & $6.6(39)$ & $6.5(30)$ & $7.3(9)$ & \\
\hline Bridging fibrosis & $0.8(5)$ & $0.6(3)$ & $1.6(2)$ & \\
\hline Definite NASH, \% (n) & $33.7(202)$ & $29.9(142)$ & $48.4(60)$ & $<0.001$ \\
\hline Borderline NASH, \% (n) & $25.4(152)$ & $26.9(128)$ & $19.3(24)$ & \\
\hline No-NASH, \% (n) & $40.9(245)$ & $43.2(205)$ & $32.3(40)$ & \\
\hline
\end{tabular}

Simple size, $n=599$. Data are expressed as relative percentages (absolute percentages are reported in parenthesis).

Children/adolescents with prediabetes or diabetes were combined into a single category, because the number of children with diabetes was small $(n=5)$. Differences between two groups were tested by the Fisher's exact test. 
Table 3. Association between prediabetes/diabetes status and risk of histologic NASH in children/adolescents with biopsy-proven NAFLD.

\begin{tabular}{|l|l|l|l|}
\hline & $\begin{array}{l}\text { Odds } \\
\text { Ratio(s) }\end{array}$ & $\begin{array}{l}\mathbf{9 5 \%} \text { Confidence } \\
\text { Interval(s) }\end{array}$ & P value \\
\hline Unadjusted model & & & \\
\hline Normal glucose tolerance & Ref. & Ref. & \\
\hline Prediabetes/diabetes status (yes vs. no) & 2.19 & $1.47-3.29$ & $<0.001$ \\
\hline Adjusted model 1 & & & \\
\hline Normal glucose tolerance & Ref. & Ref. & \\
\hline Prediabetes/diabetes status (yes vs. no) & 1.69 & $1.06-2.69$ & 0.032 \\
\hline Age (years) & 1.09 & $1.01-1.18$ & 0.024 \\
\hline Sex (male vs. female) & 0.76 & $0.51-1.12$ & 0.17 \\
\hline Waist circumference (cm) & 1.08 & $1.06-1.12$ & $<0.001$ \\
\hline Adjusted model 2 (supplementary analysis, $n=328)$ & & & \\
\hline Normal glucose tolerance & Ref. & Ref. & \\
\hline Prediabetes/diabetes status (yes vs. no) & 2.23 & $1.13-4.39$ & 0.021 \\
\hline Age (years) & 1.03 & $0.93-1.14$ & 0.57 \\
\hline Sex (male vs. female) & 0.88 & $0.51-1.51$ & 0.65 \\
\hline Waist circumference (cm) & 1.10 & $1.07-1.13$ & $<0.001$ \\
\hline PNPLA3 rs738409 variant & & & \\
\hline CC genotype (\%) & $R e f$. & $R e f$. & 0.001 \\
\hline GC genotype (\%) & 2.94 & $1.54-5.63$ & $<0.001$ \\
\hline GG genotype (\%) & 11.9 & $5.38-26.5$ & \\
\hline
\end{tabular}

Sample size, $n=599$, unless where indicated. Data are expressed as odds ratio and $95 \%$ confidence intervals as tested by logistic regression analysis. The dependent variable for all logistic regression models was the presence of definite NASH on histology (i.e., definite NASH vs. no-NASH/borderline NASH considered together). Presence of prediabetes and diabetes were combined into a single category, because the number of children with diabetes was small $(n=5)$.

Abbreviations: PNPLA3, patatin-like phospholipase domain-containing protein 3; Ref., reference category. 
Table 4. Associations between prediabetes/diabetes status and risk of different histological features of NAFLD in children/adolescents with biopsy-proven NAFLD.

\begin{tabular}{|c|c|c|c|}
\hline & Odds Ratio(s) & 95\% Confidence Interval(s) & $\begin{array}{l}P \\
\text { value }\end{array}$ \\
\hline \multicolumn{4}{|l|}{ Severe hepatic steatosis } \\
\hline \multicolumn{4}{|l|}{ Unadjusted model } \\
\hline Normal glucose tolerance & Ref. & Ref. & \\
\hline Prediabetes/diabetes status (yes vs. no) & 2.00 & $1.29-3.12$ & $<0.005$ \\
\hline \multicolumn{4}{|l|}{ Adjusted model 1} \\
\hline Normal glucose tolerance & Ref. & Ref. & \\
\hline Prediabetes/diabetes status (yes vs. no) & 1.63 & $1.02-2.62$ & 0.042 \\
\hline Age (years) & 0.97 & $0.89-1.06$ & 0.55 \\
\hline Sex (male vs. female) & 0.84 & $0.56-1.27$ & 0.42 \\
\hline Waist circumference $(\mathrm{cm})$ & 1.06 & $1.04-1.08$ & $<0.001$ \\
\hline \multicolumn{4}{|l|}{ Severe lobular inflammation } \\
\hline \multicolumn{4}{|l|}{ Unadjusted model } \\
\hline Normal glucose tolerance & Ref. & Ref. & \\
\hline Prediabetes/diabetes status (yes vs. no) & 2.26 & $1.39-3.68$ & $<0.005$ \\
\hline \multicolumn{4}{|l|}{ Adjusted model 1} \\
\hline Normal glucose tolerance & Ref. & Ref. & \\
\hline Prediabetes/diabetes status (yes vs. no) & 1.66 & $0.99-2.81$ & 0.053 \\
\hline Age (years) & 1.13 & $1.03-1.24$ & 0.012 \\
\hline Sex (male vs. female) & 0.78 & $0.48-1.26$ & 0.30 \\
\hline Waist circumference $(\mathrm{cm})$ & 1.06 & $1.03-1.08$ & $<0.001$ \\
\hline \multicolumn{4}{|l|}{ Hepatic ballooning } \\
\hline \multicolumn{4}{|l|}{ Unadjusted model } \\
\hline Normal glucose tolerance & Ref. & Ref. & \\
\hline Prediabetes/diabetes status (yes vs. no) & 1.54 & $0.97-2.46$ & 0.069 \\
\hline \multicolumn{4}{|l|}{ Adjusted model 1} \\
\hline Normal glucose tolerance & Ref. & Ref. & \\
\hline Prediabetes/diabetes status (yes vs. no) & 1.16 & $0.69-1.94$ & 0.57 \\
\hline Age (years) & 1.03 & $0.94-1.12$ & 0.54 \\
\hline Sex (male vs. female) & 0.69 & $0.45-1.08$ & 0.11 \\
\hline Waist circumference $(\mathrm{cm})$ & 1.06 & $1.04-1.08$ & $<0.001$ \\
\hline \multicolumn{4}{|c|}{ Significant fibrosis (i.e., periportal or bridging fibrosis) } \\
\hline \multicolumn{4}{|c|}{\begin{tabular}{l|l|l} 
Unadjusted model & \\
\end{tabular}} \\
\hline Normal glucose tolerance & Ref. & Ref. & \\
\hline Prediabetes/diabetes status (yes vs. no) & 1.30 & $0.64-2.66$ & 0.45 \\
\hline \multicolumn{4}{|l|}{ Adjusted model 1} \\
\hline Normal glucose tolerance & Ref. & Ref. & \\
\hline Prediabetes/diabetes status (yes vs. no) & 1.05 & $0.49-2.24$ & 0.89 \\
\hline Age (years) & 0.89 & $0.78-1.02$ & 0.10 \\
\hline Sex (male vs. female) & 0.90 & $0.48-1.68$ & 0.73 \\
\hline Waist circumference $(\mathrm{cm})$ & 1.06 & $1.03-1.09$ & $<0.001$ \\
\hline
\end{tabular}

Sample size, $n=599$. Data are expressed as odds ratio and $95 \%$ confidence intervals as tested by logistic regression analysis.

Abbreviations: PNPLA3, patatin-like phospholipase domain-containing protein 3; Ref., reference category. 
FIGURE LEGEND

Fig. 1. Prevalence of normal glucose tolerance, prediabetes and diabetes in a cohort of 599 children and adolescents with biopsy-proven NAFLD, stratified by sex. 